\begin{tabular}{|c|c|}
\hline Citation & $\begin{array}{l}\text { W. Mathues, C. Mcllroy, O.G. Harlen and C. Clasen, } \\
\text { Capillary breakup of suspensions near pinch-off, } \\
\text { Physics of Fluids } 27 \text { (9), } 093301 \text { (2015) }\end{array}$ \\
\hline Archived version & $\begin{array}{l}\text { Publishers version } \\
\text { Copyright } 2015 \text { American Institute of Physics. This article may be } \\
\text { downloaded for personal use only. Any other use requires prior permission of } \\
\text { the author and the American Institute of Physics. }\end{array}$ \\
\hline Published version & $\begin{array}{l}\text { The following article appeared in The following article appeared Physics of } \\
\text { Fluids } 27 \text { (9) and may be found at and may be found at } \\
\text { http://dx.doi.org/10.1063/1.4930011 }\end{array}$ \\
\hline Journal homepage & http://scitation.aip.org/content/aip/journal/pof2 \\
\hline Author contact & $\begin{array}{l}\text { christian.clasen@cit.kuleuven.be } \\
+32(0) 16322354\end{array}$ \\
\hline IR & $\begin{array}{l}\text { https://lirias.kuleuven.be/bitstream/123456789/495139/5/Mathues-POF-2015- } \\
\text { CapillaryBreakupSuspensions.pdf }\end{array}$ \\
\hline
\end{tabular}

(article begins on next page) 


\section{AIP $\left.\right|_{\text {Physics of }}$ Fluids}

\section{Capillary breakup of suspensions near pinch-off}

Wouter Mathues, Claire Mcllroy, Oliver G. Harlen, and Christian Clasen

Citation: Physics of Fluids 27, 093301 (2015); doi: 10.1063/1.4930011

View online: http://dx.doi.org/10.1063/1.4930011

View Table of Contents: http://scitation.aip.org/content/aip/journal/pof2/27/9?ver=pdfcov

Published by the AIP Publishing

\section{Articles you may be interested in}

Super-diffusion in sheared suspensions

Phys. Fluids 27, 041705 (2015); 10.1063/1.4918613

Jetted mixtures of particle suspensions and resins

Phys. Fluids 26, 101701 (2014); 10.1063/1.4897238

Modelling capillary break-up of particulate suspensions

Phys. Fluids 26, 033101 (2014); 10.1063/1.4866789

Microbubble formation and pinch-off scaling exponent in flow-focusing devices

Phys. Fluids 23, 092001 (2011); 10.1063/1.3631323

The effect of inlet conditions on concentrated suspension flows in abrupt expansions Phys. Fluids 18, 123303 (2006); 10.1063/1.2409619

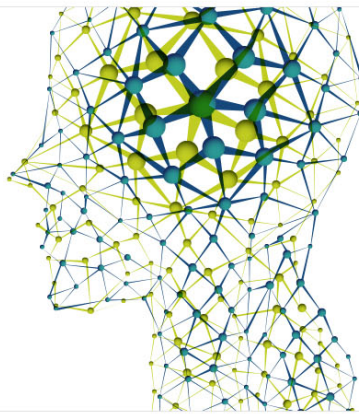




\title{
Capillary breakup of suspensions near pinch-off
}

\author{
Wouter Mathues, ${ }^{1}$ Claire Mcllroy, ${ }^{2}$ Oliver G. Harlen, ${ }^{2}$ \\ and Christian Clasen ${ }^{1, a)}$ \\ ${ }^{1}$ Department of Chemical Engineering, University of Leuven (KU Leuven), Celestijnenlaan \\ 200F, 3001 Heverlee, Belgium \\ ${ }^{2}$ Department of Applied Mathematics, University of Leeds, Woodhouse Lane, \\ Leeds LS2 9JT, United Kingdom
}

(Received 25 March 2015; accepted 12 August 2015; published online 9 September 2015)

\begin{abstract}
We present new findings on how the presence of particles alters the pinch-off dynamics of a liquid bridge. For moderate concentrations, suspensions initially behave as a viscous liquid with dynamics determined by the bulk viscosity of the suspension. Close to breakup, however, the filament loses its homogeneous shape and localised accelerated breakup is observed. This paper focuses on quantifying these final thinning dynamics for different sized particles with radii between $3 \mu \mathrm{m}$ and $20 \mu \mathrm{m}$ in a Newtonian matrix with volume fractions ranging from 0.02 to 0.40 . The dynamics of these capillary breakup experiments are very well described by a one-dimensional model that correlates changes in thinning dynamics with the particle distribution in the filament. For all samples, the accelerated dynamics are initiated by increasing particle-density fluctuations that generate locally diluted zones. The onset of these concentration fluctuations is described by a transition radius, which scales with the particle radius and volume fraction. The thinning rate continues to increase and reaches a maximum when the interstitial fluid is thinning between two particle clusters. Contrary to previous experimental studies, we observe that the final thinning dynamics are dominated by a deceleration, where the interstitial fluid appears not to be disturbed by the presence of the particles. By rescaling the experimental filament profiles, it is shown that the pinching dynamics return to the self-similar scaling of a viscous Newtonian liquid bridge in the final moments preceding breakup. (C) 2015 AIP Publishing LLC. [http://dx.doi.org/10.1063/1.4930011]
\end{abstract}

\section{INTRODUCTION}

Capillary-driven breakup of a liquid jet into droplets is omnipresent in daily life and is of tremendous importance in a variety of industrial applications, such as spraying, inkjet printing, and dosing operations in food or pharmaceutical industry. Fluids used in these applications often exhibit a complex rheology, making the prediction of these processes non-trivial. ${ }^{1-3}$ One reason for such divergent flow behaviour can be the presence of particles in these fluids. For example, inks used in various conventional printing operations contain solid particles that can disturb the normal jetting process. ${ }^{4,5}$ Furthermore, inkjet printing is used as a fabrication tool for ceramic components and printed electronics, where the functional components of the ink, the solid particles, are present in high concentration. ${ }^{6,7}$ Despite the practical importance of particle-laden drops, knowledge of the effects of particles on the stability and breakup of liquid jets is rather limited compared to Newtonian fluids.

The formation of droplets from a liquid jet has fascinated scientists for centuries. The study of the breakup dynamics of Newtonian fluids gained momentum after the characterisation of a pendant water drop using high-speed photography by Peregrine et al. ${ }^{8}$ In the instances preceding breakup, the drop is connected to the nozzle with a slender liquid filament that ultimately ruptures near the surface of the forming drop rather than in the centre of the filament. Moreover, this strong up-down asymmetry at the breakup point is independent of the employed setup (dripping faucet,

a) christian.clasen@cit.kuleuven.be 
continuous jet, or unstable liquid bridge), which suggests the existence of a universal solution for these free-surface flows. These findings have been extended to more viscous liquids and the effects of viscosity on the shape of the fluid thread are quantified..$^{9,10}$

The first step to discovering an analytical solution is to simplify the three-dimensional NavierStokes equations using a slenderness assumption. When the fluid thread is long and thin, the flow is predominantly directed along the axis, so the velocity field is essentially one-dimensional. By expanding the velocity field in the radial direction and taking only the leading-order terms into account, a one-dimensional version of the momentum equations is deduced. ${ }^{11,12}$ Since the flow is without a typical length scale near breakup, the concept of self-similarity can be successfully applied to this problem to retrieve an analytical solution. ${ }^{13,14}$ The length scale of this solution is time-dependent, implying that solutions at different times are identical after a correct rescaling of the filament radius and the axial coordinate.

Different self-similar solutions have been established depending on the relative importance of inertial and viscous term in the simplified Navier-Stokes equation. The dominant material parameter resisting the breakup is revealed by the Ohnesorge number $\mathrm{Oh}$, which compares the time scales of viscosity controlled breakup and inertia controlled breakup and is defined as

$$
O h=\frac{\eta}{\sqrt{\rho \gamma R}}
$$

where $\eta$ is the viscosity, $\rho$ is the fluid density, $\gamma$ is the surface tension, and $R$ is the relevant length scale, e.g., the jet radius. For moderate Ohnesorge numbers, a universal solution considering all terms of the one-dimensional Navier-Stokes equation has been developed by Eggers. ${ }^{15}$ In this case, the minimum radius observed along the jet, $R_{m}$, obeys the thinning law

$$
R_{m}=0.0304 \frac{\gamma}{\eta}\left(t_{p}-t\right),
$$

where $t_{p}$ is the pinching time, where the jet breaks. Although the universality of the solution implies a validity for all fluids, in practice, this inertia-viscous (IV) scaling is only observed in close proximity to breakup for fluids in a narrow viscosity range. ${ }^{16}$ Nevertheless, Newtonian fluids with these viscosities are employed to generate stable droplets, for example, in inkjet printing applications. ${ }^{7}$ More viscous fluids with larger Ohnesorge numbers tend to follow a different, viscous (V) scaling. The inertial term may be dropped in this case, reducing the momentum equation to the Stokes form. A symmetric self-similar solution to this equation was derived by Papageorgiou, ${ }^{17}$ giving the following expression for the minimal radius:

$$
R_{m}=0.0709 \frac{\gamma}{\eta}\left(t_{p}-t\right)
$$

Since the fluid effectively undergoes a uniaxial extensional flow, free-surface flows are also employed in rheometry. The breakup dynamics offer insight into the extensional properties of relatively low viscous fluids, which cannot be measured with standard rheological techniques. ${ }^{18}$ The temporal evolution of the minimal radius is used in combination with the correct scaling law to extract material parameters. For Newtonian fluids, McKinley and Tripathi ${ }^{19}$ show that the viscous scaling law (Eq. (3)) can be used to extract the capillary velocity $v_{c}=\gamma / \eta$. For non-Newtonian fluids, the material properties that have been determined include the apparent extensional viscosity, the relaxation time of elastic samples, and the power law parameter for strain-thinning liquids. ${ }^{2,20-22}$ Although different experimental setups are possible, these capillary breakup experiments are mostly executed with an unstable bridge created by stretching a liquid sample between two end plates. This setup has the advantage of an Eulerian reference frame, so the evolution of the minimal diameter is easily followed with a laser micrometer or a high-speed camera.

Recently, the extensional properties of suspensions have been investigated with free-surface flows. Dispersions that exhibit shear-thickening can show viscoelastic pinch-off or dilatancy effects depending on the strain rate. ${ }^{23,24}$ However, other non-Brownian suspensions display a faster thinning rate close to pinch-off than that predicted by the viscous scaling law for the bulk viscosity. ${ }^{25-30}$ This flow behaviour is not necessarily a strain-thinning effect and can rarely be captured by a power-law model. ${ }^{30}$ The shear response of these systems is dominated by hydrodynamic 
interactions and can be described by a single viscosity up to moderate particle concentrations. ${ }^{31}$ This viscosity is proportional to the viscosity of the suspending medium and has a strong dependance on the particle volume fraction $\phi$. Careful experiments on the shear-rate dependance of these suspensions are performed by Zarraga et al. ${ }^{32}$ who found no shear thinning for $\phi<0.45$. Many semi-empirical correlations have been proposed to describe the relative shear viscosity $\eta_{r}$ of the suspension. Throughout this paper, we will use the Maron-Pierce model. ${ }^{33}$

$$
\eta_{r}(\phi)=\left(1-\frac{\phi}{\phi_{m}}\right)^{-2},
$$

where $\eta_{r}=\eta / \eta_{m}$ is the relative viscosity compared to the medium viscosity $\eta_{m}$. The highest volume fraction is estimated by the random close packing limit of $\phi_{m}=0.64 .^{34}$ This equation has the same form as the commonly used Krieger-Dougherty expression, ${ }^{35}$ which employs a different power law exponent $-[\eta] \rho \phi_{m}$, where $[\eta]$ is the intrinsic viscosity.

The acceleration that is observed during the late stages of capillary breakup of these suspensions is caused by the finite-size effects of the particles, rendering the continuum approximation invalid for small filaments. Furbank and Morris ${ }^{26,27}$ demonstrate this in an extensive experimental study on dripping and jetting flows of granular suspensions (with particle radius $R_{p}=53-103 \mu \mathrm{m}$ ) and describe the thinning as a two-stage process. The necking is initially dominated by the suspension viscosity, followed by a second stage in which the suspending fluid properties appear to be critical. Lindner and co-workers continue this work by performing dripping experiments of granular suspensions with a wide range of particle sizes and concentrations. The second stage is split into a small regime where the thinning rate is independent of the volume fraction, matching that of the medium fluid, and an accelerated regime where the suspension clearly thins faster than the medium fluid. ${ }^{25}$ This acceleration is observed at very low particle concentrations, even when there is only a single particle in the thinning filament. ${ }^{28}$

In this paper, we quantify and explain this acceleration of suspensions near pinch-off with capillary breakup tests of an unstable liquid bridge. Contrary to previous studies where the breakup dynamics are examined by comparison to viscous oils with approximately the same viscosity, we will only use the temporal evolution of the minimal filament radius of the suspensions and apply the self-similar scaling laws to extract material parameters. Furthermore, we focus on the final thinning stage and use high-resolution optics to image the fluid dynamics and distinguish the motion of individual particles. The paper is structured as follows. In Section II, the employed model suspensions and the experimental setup are introduced, as well as the one-dimensional model previously established by McIlroy and Harlen, ${ }^{36}$ which is used to simulate the thinning behaviour of the suspensions. Reference capillary breakup experiments on the Newtonian suspending media are presented in Section III. The different stages in the pinching of the suspensions are described and compared to the one-dimensional model in Section IV, where a clear distinction is made between the thinning of the suspension (Section IV B) and that of the interstitial fluid (Section IV C).

\section{MATERIALS AND METHODS}

\section{A. Experimental measurements}

The model suspensions consist of non-Brownian spherical particles dispersed in Newtonian silicone oils with volume fractions $\phi$ between 0.02 and 0.40 . The key physical properties of the poly(dimethylsiloxane) (PDMS) oils at a temperature of $22^{\circ} \mathrm{C}$ are summarised in Table I. Three different-sized particles are used: polystyrene spheres with a radius $R_{p}=10 \mu \mathrm{m}$ (PS10) and $R_{p}=$ $20 \mu \mathrm{m}$ (PS20), and poly(methyl methacrylate) spheres with $R_{p}=3 \mu \mathrm{m}$ (PMMA3) (Dynoseeds, Norway). The particles are initially dispersed in the continuous phase using a vortex mixing device (Vortex Genie 2, Scientific Industries) and the suspensions are subsequently placed on a rolling bench for $30 \mathrm{~min}$ to homogenise the samples. The PS spheres have a density of $\rho=1050 \mathrm{~kg} / \mathrm{m}^{3}$ and the PMMA spheres of $\rho=1180 \mathrm{~kg} / \mathrm{m}^{3}$.

To judge the stability of the dispersions, the sedimentation velocity $v_{s, 0}$ for dilute suspensions is calculated using Stokes' law, 
TABLE I. Characteristics of the silicone oils at a temperature of $22^{\circ} \mathrm{C}$.

\begin{tabular}{lcccc}
\hline \hline & $\gamma(\mathrm{mN} / \mathrm{m})$ & $\rho\left(\mathrm{kg} / \mathrm{m}^{3}\right)$ & $\eta_{m}(\mathrm{~Pa} \mathrm{~s})$ & $O h(-)$ \\
\hline PDMS 1 & 21.0 & 1070 & 0.180 & 1.20 \\
PDMS 2 & 21.0 & 970 & 0.360 & 2.52 \\
\hline \hline
\end{tabular}

$$
v_{s, 0}=\frac{2 R_{p}^{2} \Delta \rho g}{9 \eta_{m}},
$$

where $\Delta \rho$ is the difference between the densities of particle and fluid, and $g$ is the gravitational constant. For more concentrated suspensions, the settling velocity $v_{s}$ is calculated by the empirical Richardson-Zaki expression, ${ }^{31}$

$$
\frac{v_{s}}{v_{s, 0}}=(1-\phi)^{6.55},
$$

where the exponent is chosen to match the prediction for dilute particle concentrations by Batchelor. ${ }^{37}$ The maximal sedimentation velocity is determined for the dilute samples of PS20 to be $v_{s} \approx 10 \mu \mathrm{m} / \mathrm{min}$. Therefore, each experiment is performed within $15 \mathrm{~min}$ after (re)dispersing the particles using the vortex mixer.

Capillary breakup experiments are performed with the CaBER-1 extensional rheometer (Thermo Scientific, Germany), equipped with circular disks with a radius $R_{0}=2 \mathrm{~mm}$ and an initial gap of $2 \mathrm{~mm}$, at an ambient temperature of $22{ }^{\circ} \mathrm{C}$. Sample volumes of $0.03 \mathrm{ml}$ are loaded between the plates with a syringe through a nozzle of $1.36 \mathrm{~mm}$ (EFD Nordson Precision tips). To create an unstable liquid bridge, the samples are linearly stretched within $50 \mathrm{~ms}$ to final plate separations of 6 and $8 \mathrm{~mm}$. However, it should be noted that no change in the thinning behaviour is observed when changing the final gap. The thinning dynamics depend on the local distribution of the particles, which varies statistically between each experimental run. Therefore, experiments are repeated at least ten times to account for this statistical variation and the parameters extracted from the thinning curves are averaged over at least ten representative measurements.

The thinning and breakup dynamics are monitored with a high-speed camera (Fastcam SA2, Photron, USA) to capture the heterogeneous nature of the final breakup process of suspensions. The camera is connected to a tube lens system equipped with $5 \times$ and $10 \times$ microscopic objectives (Olympus, Japan), resulting in a spatial resolution of $1.9 \mu \mathrm{m} / \mathrm{pixel}$ and $0.95 \mu \mathrm{m} / \mathrm{pixel}$, respectively. This resolution allows the identification of individual particles in the liquid filament and enables accurate tracking of their position near breakup. Images are generally taken at a rate of $3000 \mathrm{fps}$ with a shutter time of $10 \mu \mathrm{s}$, but rates are increased to $9000 \mathrm{fps}$ for examining the final pinch-off dynamics. Illumination is achieved by a fibre-optic illuminator (Fiber-Lite DC-950, Dolan Jenner Industries, USA) and a $50 \mathrm{~mm}$ condenser lens. The image series are analysed with Matlab-based, self-written image processing routines ${ }^{38}$ in order to determine the shape of the edges of the filament and to follow the evolution of the minimal radius in time $R_{m}(t)$.

The Hencky strain $\epsilon_{H}$ and the strain rate $\dot{\epsilon}$ are determined from the radius data via

$$
\begin{gathered}
\epsilon_{H}(t)=2 \ln \left(\frac{R_{0}}{R_{m}}\right), \\
\dot{\epsilon}(t)=-\frac{2}{R_{m}} \frac{\partial R_{m}}{\partial t} .
\end{gathered}
$$

Since the required numerical differentiation for $\dot{\epsilon}$ is susceptible to noise, the filament radius data are smoothed with a Savitzky-Golay filter with a second-order polynomial and a fitting window of 21 points. This technique is preferred to weight-adjacent averaging, as it tends to better preserve features of the data.

Since micron-size particles are investigated in a relatively high-viscosity medium, Brownian motion is unable to re-distribute the particles and eliminate particle-density gradients within the time scale of the experiment. This can be verified from the value of the Péclet number $P e$, which is the ratio 
of the rate of advection by the flow to the rate of diffusion by Brownian motion in a dilute dispersion,

$$
P e=\frac{6 \pi \eta_{m} \dot{\epsilon} R_{p}^{3}}{k_{B} T},
$$

where $k_{B}$ is the Boltzmann constant and $T$ is the absolute temperature. The strain rate has the lowest value during capillary breakup experiments of the most viscous samples and a characteristic value $\dot{\epsilon} \sim 10 \mathrm{~s}^{-1}$ can be estimated by the inverse viscous time scale $\dot{\epsilon} \sim \gamma /\left(\eta R_{0}\right)$. Even for the smallest particles the Péclet number is of the order $P e=O\left(10^{4}\right)$, which justifies neglecting Brownian motion in our study.

\section{B. Simulating particulate suspensions}

To model the effects of finite particle size on the dynamics of filament thinning, we use the one-dimensional method of McIlroy and Harlen ${ }^{36}$ to examine the effects of axial variations in particle concentration. In this model, the filament generated by the CaBER is assumed to be sufficiently long and thin that it can treated as an axisymmetric slender jet. Thus, the governing equations for the dimensionless filament radius $h(z, t)$ and the velocity $v(z, t)$ are given by

$$
\begin{aligned}
\frac{\partial h^{2}}{\partial t}+\frac{\partial}{\partial z}\left(h^{2} v\right) & =0, \\
\frac{\partial}{\partial t}\left(h^{2} v\right)+\frac{\partial}{\partial z}\left(h^{2} v^{2}\right) & =\frac{\partial}{\partial z}\left(h^{2}\left(K+3 O h \frac{\partial v}{\partial z}\right)\right),
\end{aligned}
$$

from conservation of mass and momentum, respectively. Here the curvature term is defined as

$$
K=\frac{h_{z z}}{\left(1+h_{z}^{2}\right)^{3 / 2}}+\frac{1}{h\left(1+h_{z}^{2}\right)^{1 / 2}},
$$

with the $z$-subscript denoting differentiation with respect to $z$. These governing equations (Eqs. (10) and (11)) have been non-dimensionalised with respect to the initial radius $R_{0}$ and the Rayleigh time scale $t_{R}=\sqrt{\rho R_{0}^{3} / \gamma}$. The initial shape of the free surface is modelled as an arc of a circle to simulate a liquid bridge held between two end plates, as in the CaBER technique.

For each realisation, the particles are initially uniformly distributed at random axial positions $z_{p}$, for $p=1, \ldots, N_{p}$, throughout the liquid bridge. The average volume fraction is given by

$$
\phi_{a v}=\frac{N_{p}}{V_{t o t}} V^{p}
$$

where $V^{p}$ is the particle volume, which for spherical particles is

$$
V^{p}=\frac{4}{3} \pi R_{p}^{3}
$$

Thus, the effective particle radius $R_{p}$ is controlled by varying the number of particles $N_{p}$. Also for the numerical calculations, the particles are assumed to be sufficiently large that Brownian motion is negligible. In addition, we assume that the overall effects of particle-particle and particle-surface interactions can be neglected so that each particle moves with the axial velocity $v\left(z_{p}, t\right)$ of the fluid, obtained by linear interpolation. The local volume fraction $\phi$ is then determined from the number of particles within a segment of the filament. This concentration discretisation is larger than the mesh size used in the solution of Eq. (10) and, within limits, does not affect the ultimate thinning dynamics. ${ }^{36}$

The effects of particle concentration on the dynamics are incorporated by assigning a local viscosity from the Maron-Pierce relation (see Eq. (4)), which introduces a local Ohnesorge number in Eq. (10), given by

$$
O h_{i}=O h_{s}\left(1-\frac{\phi_{i}}{\phi_{\max }}\right)^{-2}, \quad i=1, \ldots, N_{b},
$$

where $O h_{s}$ denotes the Ohnesorge number of the solvent and $N_{b}$ represents the number of bins into which the filament is divided for the concentration discretisation. In this way, the particles contribute 
only to the local viscosity of the fluid and the direct effects of individual particles on the free surface are neglected. The governing equations (Eqs. (10) and (11)) are then solved via a semi-implicit numerical scheme, where the velocity terms are treated explicitly. Simulations are executed to mimic the particles used in the experiments for various concentrations $\phi<0.25$. In general and similar to the experimental protocol, ten realisations are performed for a single suspension to account for the statistical variation arising from the initial particle distribution. In the case of $R_{p}=3 \mu \mathrm{m}$, a single realisation is executed for $\phi=0.02$ and $\phi=0.10$ due to computational time constraints.

\section{NEWTONIAN OILS}

As reference cases, capillary breakup experiments are performed on the two silicone oils without particles. For these inelastic fluids, the dominant material parameter resisting the breakup is the Ohnesorge number. ${ }^{2}$ The global Ohnesorge number for this experiment given in Table I uses $R=R_{0}\left(L_{1} / L_{0}\right)^{-3 / 4}$ in Eq. (1), which is the radius predicted at the cessation of stretching, determined by a lubrication solution for a Newtonian viscous fluid. ${ }^{39}$ By comparing the characteristic thinning velocities of the viscosity and inertia dominated regimes, the boundary between the two regimes is located at a critical value $O h^{*}=0.2077 .{ }^{16}$ Since the values in Table I are sufficiently above $O h^{*}$, both oils initially display viscosity-dominated filament thinning.

Figure 1 shows the evolution of the minimal radius during capillary breakup of the two silicone oils, in which the pinching time $t_{p}$ is used as a temporal reference. As expected from the value of the Ohnesorge number, which is greater than $\mathrm{Oh}^{*}$, both fluids thin linearly in time consistent with the viscous scaling (Eq. (3)), allowing the extraction of viscosities from the thinning data. The quality of the fits over a radius range from 250 to $50 \mu \mathrm{m}$ is demonstrated in Figure 1 and the extracted viscosities $\eta_{f i t}=364 \mathrm{mPa}$ s and $\eta_{f i t}=171 \mathrm{mPa}$ s, respectively, are in agreement with the shear-viscosity values (see Table I).

However, deviations from this viscous scaling occur close to breakup. This observation is highlighted in Figure 2, where, in order to simplify the comparison between samples, we eliminate viscosity from Eqs. (2) and (3) by rescaling the time with the viscous time scale ${ }^{2}$

$$
t_{\eta}=\frac{14.1 \eta_{m} R_{0}}{\gamma}
$$

The time axis is further shifted with $t_{c}$, the time at which the filament radius equals the reference radius. As a result, the thinning curves of both samples initially coincide, following the viscous scaling (Eq. (3)) until a radius $R_{m}=50 \mu \mathrm{m}$. Although the filament thinning of PDMS 2 follows the

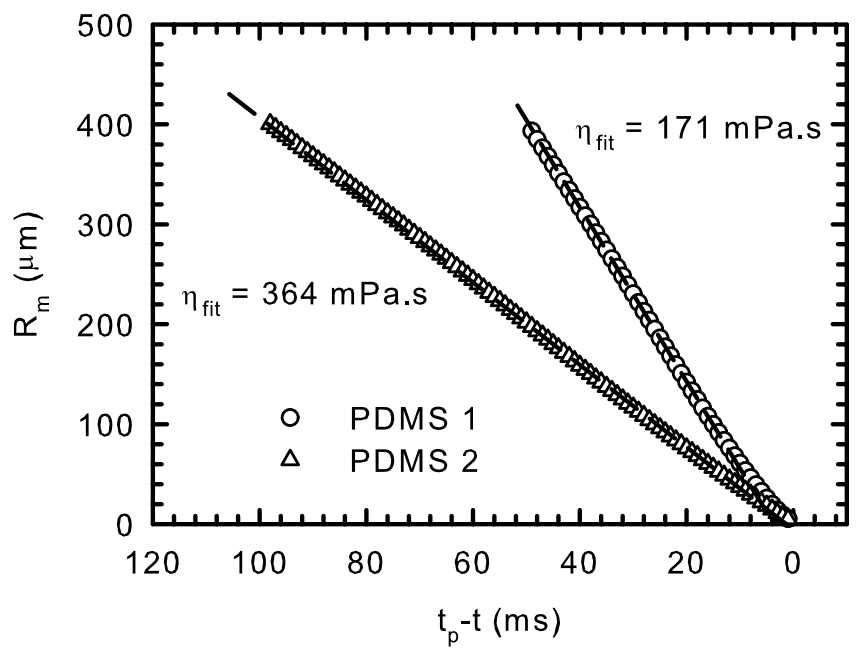

FIG. 1. Thinning dynamics of the two viscous silicone oils. Both oils follow the viscous scaling for the most part of breakup process. 


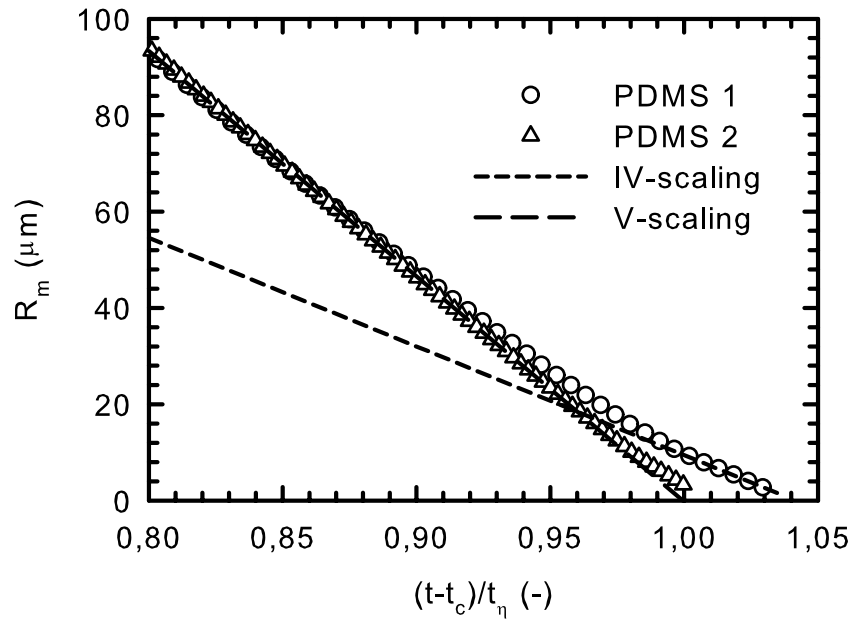

FIG. 2. Enlargement of the final thinning dynamics of both silicone oils. The time is rescaled with $t_{\eta}$ to allow a better comparison of both samples. The low viscous PDMS 1 clearly exhibits a transition from the viscous (V) scaling to the inertia-viscous (IV) scaling during the final breakup stages.

viscous scaling until breakup, the thinning of the less viscous PDMS 1 undergoes a transition and decelerates prior to breakup.

This transition in scaling has been explained by Eggers. ${ }^{13,15}$ He shows that the decaying filament radius gives rise to a strongly increasing capillary pressure close to pinch-off, resulting in a diverging strain rate (see Eq. (8)). Hence, the inertial term grows more quickly than the other terms and can no longer be neglected in the dominant balance. Thus, the pinching of the filament is controlled by an inertia-viscous-capillary balance (IV-scaling) and follows the similarity solution described by Eq. (2) prior to breakup, rather than the V-scaling. This deceleration of the breakup dynamics is accompanied by a shift of the location of the minimum radius in the axial direction from the centre of the filament towards the end drops, turning the initial symmetric viscous pinching into an asymmetric pinching. Theoretically, the transition radius from viscous scaling to inertia-viscous scaling is approximated by equating the estimates of the inertial and capillary term as

$$
R=R_{0} O h^{2 /(2 \beta-1)},
$$

with $\beta=0.175 .{ }^{14}$ However, this value is an order of magnitude larger than our experimental transition radius, which is comparable to other experimental observations. ${ }^{40}$ The reason for this discrepancy is presently not understood, although it might be related to breaking of the symmetry which could delay the transition. ${ }^{2,41}$

Since the thinning of Newtonian fluids is generally used as a benchmark for more complex suspension dynamics, recognising this transition is crucial. Moreover, the transition radius we observe is comparable to the particle size in the current investigation and therefore comparable to the reported length scale at which changes in the breakup process occur due to finite-size effects. ${ }^{25,26}$ Thus, in order to decouple the transition of V- to IV-scaling from the suspension dynamics, most suspension experiments in this paper are performed with PDMS 2 as a continuous phase.

\section{SUSPENSIONS}

\section{A. Thinning stages}

In order to quantify the particulate effects on the pinching dynamics, we have examined the filament evolution for suspensions with varying volume fraction and particle size. As an example of the general thinning dynamics we observe, Figure 3 shows the experimental breakup curve (points) compared to the results of the numerical simulation (solid line) for $10 \%$ of PS20 in PDMS 2. We also include a series of experimental images to illustrate how the unstable filament approaches 


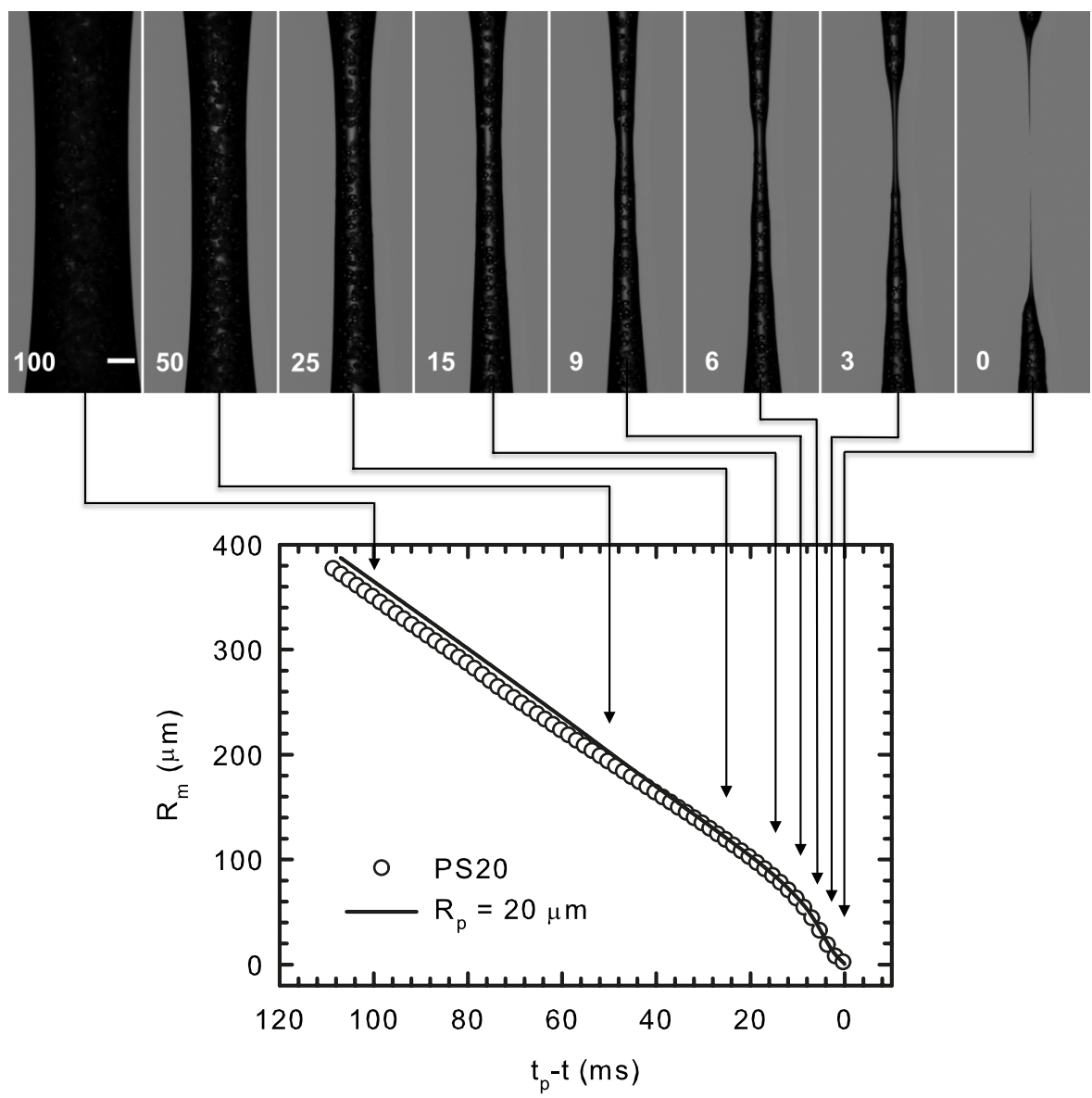

FIG. 3. Evolution of the minimal radius during capillary breakup of suspension with $\phi=0.10$ of PS20 in PDMS 2. The solid line shows the results of a simulation with the same concentration and particle size. Initially, the dynamics are solely determined by the effective viscosity of the suspension. Subsequently, the thinning velocity accelerates causing a faster rupture of the filament. The number in the bottom left of the images indicates the time to pinch-off $\left(t_{p}-t\right)$ in $\mathrm{ms}$ and the scale bar represents $200 \mu \mathrm{m}$. In the last three frames, localised thinning of the continuous phase between two particles is observed.

breakup. In general, we observe four distinct thinning regimes; a suspension regime, a concentration fluctuation regime, an accelerated regime, and a final deceleration regime governed by the properties of the medium fluid only, which we now describe.

In the initial suspension regime-illustrated by the first two images in Figure 3-the filament appears entirely black, because the particles render the sample opaque. The suspension can be considered as a homogeneous fluid and the thinning dynamics are fully determined by the effective viscosity of the suspension. The viscosity that is recovered by fitting the viscous scaling (Eq. (3)) to the minimal radius data, corresponds to the prediction of the Maron-Pierce correlation (see Eq. (4)) for a particle fraction $\phi=0.10$. In previous dripping studies, a clear linear decrease of the minimal radius $R_{m}$ with time - as predicted by the similarity solutions-is not observed. The breakup dynamics are instead interpreted by experimentally comparing the suspension breakup dynamics with the thinning of a Newtonian oil with the same viscosity as the suspension. ${ }^{25,28}$

We recognise two reasons why the study of the breakup starting from an unstable filament has a more straightforward interpretation than a dripping experiment. First, the self-similar solutions are only applicable if the slenderness assumption is valid. ${ }^{16}$ The instant creation of a slender, unstable filament in the CaBER allows the viscous scaling to remain suitable for most of the breakup process. In the dripping setup, the filament only gradually achieves the required slenderness to observe the self-similar shapes. This formation exhibits different dynamics, which are only empirically captured for suspensions. ${ }^{27}$ Second, once this filament of the dripping experiment is slender enough, 
the filament and particle radius are often of the same magnitude, particularly for larger granular particles. Hence, the fluid can no longer be treated as a continuum.

As the filament becomes thinner, transparent zones emerge and individual particles are clearly distinguished. The suspension appears heterogeneous revealing lighter oil-rich regions and darker zones with clusters of particles. We call this phase the concentration fluctuation regime. Regions of the filament with a lower particle density locally exhibit a smaller effective viscosity and will therefore thin faster. As the model of McIlroy and Harlen ${ }^{36}$ predicts, these fluctuations in the local concentration of particles are amplified as the volume of the filament region is reduced, inducing an accelerated thinning rate compared to that of the suspension regime. Consequently, we observe a deviation from the viscous scaling law once the filament radii has reduced to $100 \mu \mathrm{m}$, as shown in Figure 3.

Despite the simplicity of the model, the corresponding simulation, shown by the solid line in Figure 3, not only captures the qualitative features of the experiments with a transition to an accelerated thinning of the filament due to fluctuations in the local particle density but it also describes quantitatively both the radius of onset and the thinning velocity during this phase of thinning. The small difference in the slope of the initial linear regime is caused by a small difference between the experimentally observed suspension viscosity and the predictions of Maron-Pierce model used in the simulations. The degree of agreement between the simulations and experiments strongly suggests that the acceleration is a consequence of the amplification of particle-density fluctuations due to thinning creating local regions of lower than average viscosity that are able to thin more easily, producing a more rapid thinning in these regions.

Particle-density fluctuations also manifest in the shape of the filament shown in Figure 3, starting from the fourth image (with time stamp 15). Unlike the previous images, we now observe local variations in the filament diameter, indicating the onset of a localised thinning. These concentration fluctuations during capillary breakup of the suspensions are also observed by Roché et al. ${ }^{24}$ during extensional experiments on concentrated cornstarch dispersions. However, the nearly periodic surface fluctuations between solvent-rich and jammed regions that are present in their work are not detected in our samples, which are significantly less concentrated. Similar concentration fluctuations have also been observed for concentrated suspensions of colloidal particles in a complex flow field with a strong extensional contribution. ${ }^{42}$

After the concentration fluctuation regime, where a certain amount of particles are still present in the thinnest region of the filament, particle-density fluctuations in the thinning filament eventually lead to particle-free sections, which are clearly observed in the last three images of Figure 3. In this particle-free zone, the radial velocity is locally accelerated, resulting in confined thinning with the filament resembling the shape of a power law fluid. ${ }^{43}$ This continuous-phase thinning begins at roughly $7 \mathrm{~ms}$ before pinch-off for this particular suspension and the evolution of the minimum radius in this phase is highlighted in Figure 4. We split this continuous phase into two distinct regimes.

First, there is an accelerated thinning regime ${ }^{25,28}$ where the radius decays linearly with time, in quantitative agreement with the model results, and the radial velocity $v_{r}=-d R_{m} / d t$ reaches a maximum. For this short period, the thinning is approximately twice as fast as that of the pure medium fluid. Enlarged pictures of this accelerated pinching stage are shown in Figure 5, in which the two nearest particles are accentuated with a grey circle. The displacement of these particles in the consecutive frames demonstrates the acceleration in this zone. In these pictures, two particle clusters appear to form a barrier for the continuous phase, isolating roughly $2 \mathrm{nl}$ of the medium fluid from the rest of the filament. Due to the slower or even arrested thinning in the clusters, the continuous phase in between is forced to resemble an hour glass shape similar to a small-scale filament stretching experiment with the clusters as boundaries, resulting in a faster pinching of the thread.

Second, the radial velocity reduces and the thinning decelerates prior to breakup. This deceleration was not detected in previous studies on similar systems ${ }^{25-29}$ because these small filament radii were below the resolution limit of the employed camera setup. By fitting these last radii with the viscous scaling (Eq. (3)), the viscosity of the interstitial viscous oil is recovered. The thinning appears to return to the self-similar scaling of a Newtonian liquid bridge, indicating that the thinning is no longer affected by the presence of the particles. This final stage where the thinning-rate of the pure suspending fluid is recovered is also captured in the simulations. 


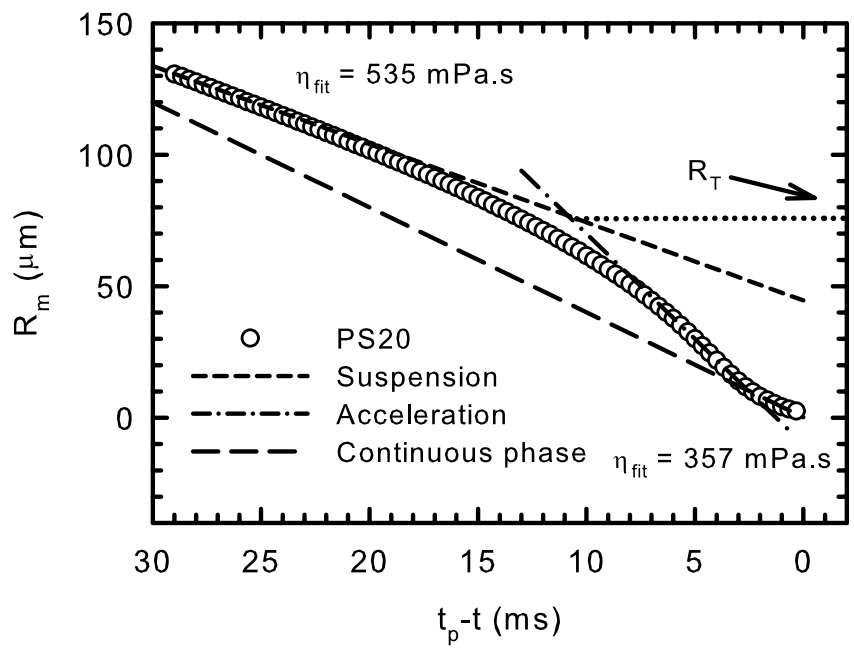

FIG. 4. Plot showing the final thinning dynamics of 10\% PS20 in PDMS 2 near pinch-off. The accelerated regime is followed by a deceleration where the dynamics appear to return to the self-similar scaling of the continuous phase, demonstrated by the fitted viscosity. The onset of suspension dilution is also indicated with the transition radius $R_{T}$.

Reviewing the breakup dynamics of this suspension, we have not encountered the interstitial fluid regime described by Bonnoit et al. ${ }^{25}$ where thinning dynamics observed between the suspension regime and the accelerated regime is governed only by the medium fluid. Figure 4 indicates that part of the concentration fluctuation stage has approximately the same thinning rate as the final regime, so this region was probably labelled the interstitial fluid regime. However, we presume that this zone is merely a transition state in the concentration fluctuation regime, because there is no distinct physical reason why the suspension would thin at this rate. In Secs. IV B and IV C, the different thinning stages are separately regarded and the effect of changing different suspension properties is investigated. We first consider the first two stages, where there are still particles present in the neck, before continuing to the thinning of the interstitial fluid in the final two stages.

\section{B. Suspension thinning and dilution}

To obtain a better understanding of the mechanisms causing this accelerated breakup, we have performed a series of CaBER experiments for different particle concentrations and sizes. We have also simulated the breakup of these suspensions with $O h_{s}=2.5$ corresponding to PDMS 2 (see Table I). Qualitatively, all samples follow the thinning curve of Figure 4, with the four thinning regimes that are described in Sec. IV A. Initially, the examined suspensions behave as an effective Newtonian fluid. The initial linear section of the thinning curve is fitted with the viscous scaling law
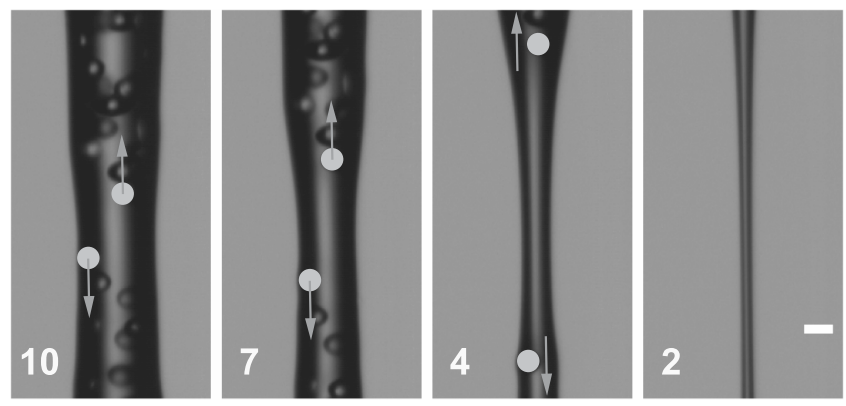

FIG. 5. Magnified image of the final stages of the capillary breakup of $10 \%$ PS20 in PDMS 2 . The number in the bottom left indicates the time to pinch-off $\left(t_{p}-t\right)$ in $\mathrm{ms}$ and the scale bar represents $50 \mu \mathrm{m}$. The final thinning takes place between two particles which are highlighted by the grey circles and their displacement reveals the large extension rates before pinch-off. 


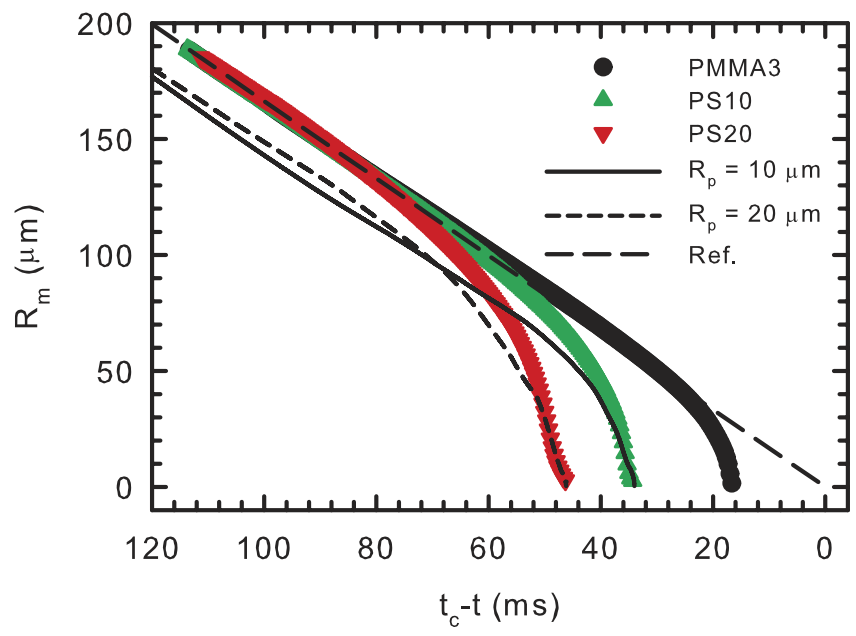

FIG. 6. Breakup dynamics of suspensions of different particle sizes in PDMS 2 with a fixed volume fraction $\phi=0.25$. The symbols show the experiments and the solid lines the simulated thinning profiles for the two largest particles. The time axis is shifted to demonstrate that the initial effective viscosity regime is independent of particle size. Breakup occurs considerably sooner for the suspensions with the larger particles. As reference, the radius evolution of a viscous fluid with the same viscosity is included as a dashed line.

(Eq. (3)) for each sample to acquire the characteristic viscosity. The extracted viscosity is independent of particle size and the concentration dependance is well described by the model prediction for the suspension viscosity (Eq. (4)).

As the filament becomes thinner, particle-density fluctuations are amplified for all examined samples. Local heterogeneities consequently emerge and the fastest thinning occurs in a locally diluted region. Although each sample demonstrates the four thinning regimes, we observe differences in the thinning rate of the accelerated regime and the transitions between the consecutive thinning stages occur at different filament radii. By examining the local slopes of the thinning curves, we explore the effects that changing suspension properties have on the concentration fluctuation regime.

Figure 6 shows the evolution of the minimal filament radius for three suspensions of different particles with a fixed volume fraction $\phi=0.25$. The results of the one-dimensional model are shown by the three lines. Initially, the thinning trajectories of the three samples overlap in the effective fluid stage, as the suspension viscosity depends solely on the volume fraction. However, the suspension with largest particles deviates from this linear thinning much earlier, so that while the smallest particle (PMMA3) suspension is still in the first thinning stage, the PS20 sample has already pinched due to a combination of the local suspension dilution and the accelerated thinning of the interstitial fluid. Although the model results are in qualitative agreement with the experimental data and represent the final thinning dynamics well, we observe differences in the radii at which the thinning departs from the initial suspension regime, indicating the onset of concentration fluctuations.

Similarly, Figure 7 shows the evolution of the minimal filament radius for three suspensions of PS40 having volume fractions $\phi=0.02,0.10$, and 0.25 . Again, the model results are given by the lines. In this case, the time axis is rescaled by $t_{\eta}$ (Eq. (15)) so that, as in Figure 6, the initial thinning trajectory of the three samples overlap. A deviation from the linear suspension regime is observed at larger filament radii for the more concentrated experimental samples and the model results are in qualitative agreement. Again, we observe small differences in the concentration fluctuation radius predicted by the model compared to the experimental data.

We have chosen to quantify the onset of the dilution with a transition radius $R_{T}$, which is defined as the intersection between fitting lines of the suspension regime and the accelerated regime (see Figure 4). The precise value of the transition radius depends upon the initial particle distribution and so varies in consecutive experiments and numerical realisations of the same suspension. However, in general, the average transition radius increases with particle size, as seen in Figure 6, 


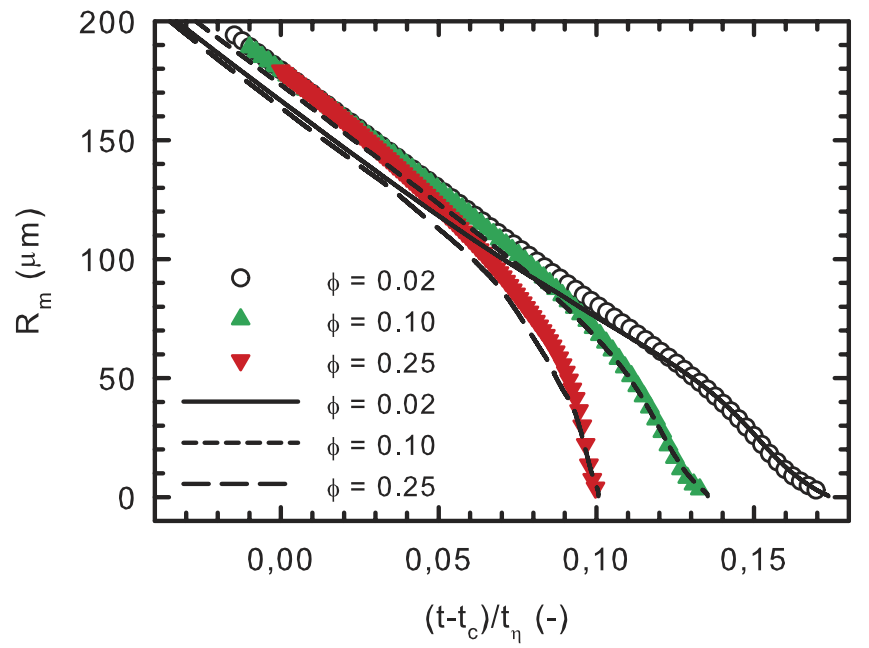

FIG. 7. Breakup dynamics of PS40 in PDMS 2 with volume fractions $\phi=0.02,0.10$, and 0.25 . The symbols show the experiments and the solid lines the simulated thinning profiles. The time axis is shifted and rescaled with $t_{\eta}$ for better comparison of the three samples.

and volume fraction, as seen in Figure 7. In order to understand this evolution, and the differences between the experiments and the model behaviour, we define a structural length scale $R_{s}$ for the variation in the particle density.

The distribution of non-interacting, identical spherical particles in a homogeneous suspension is characterised statistically by the particle nearest-neighbour probability density function $H_{p}(r),{ }^{44}$ the probability associated with finding the nearest-neighbour particle at some given distance $r$ from a reference particle. For an ideal system of non-interacting point particles, this function is given by ${ }^{45}$

$$
H_{p}(r)=4 \pi \rho_{N} r^{2} \exp \left(-\frac{4}{3} \pi \rho_{N} r^{3}\right)
$$

where the particle number density $\rho_{N}$ can be expressed in terms of the volume fraction and the particle radius as $\rho_{N}=3 \phi /\left(4 \pi R_{p}^{3}\right)$. Thus, a length scale for concentration variations can be obtained as the standard deviation of the nearest-neighbour distance $\sqrt{\left\langle r^{2}\right\rangle}$, obtained from the second central moment of $H_{p}(r)$. However, it is only when these concentration variations lead to a significant change in fluid viscosity that they affect the thinning dynamics. To account for this effect, we multiply $\sqrt{\left\langle r^{2}\right\rangle}$ with the derivative of the logarithm of the suspension viscosity with respect to volume fraction. Using the Maron-Pierce model (Eq. (4)), the length scale $R_{S}$ for the transition radius is defined as

$$
R_{s}=\sqrt{\left\langle r^{2}\right\rangle}\left(\frac{1}{\eta} \frac{\partial \eta}{\partial \phi}\right)=\frac{0.65 R_{p}}{\phi^{1 / 3}\left(\phi_{m}-\phi\right)} .
$$

In Figure 8, we use this to rescale the transition radii of various suspensions. The scaling works well for the simulated transition radii, particularly in the range $\phi=0.10-0.25$. For smaller concentrations, the transition between the suspension regime and the accelerated regime is not as pronounced, as the slopes of the respective regimes are similar. However, despite having qualitatively the correct features of the experiments, the length scale $R_{s}$ does not capture the lower transition radii at low volume fractions and overestimates the transition radii at high concentrations $(\phi=0.40)$. It is to be expected that this scaling should work better for the simulated thinning profiles than the experiments as both neglect the effects of the excluded volume in the particle distributions, whereas the experimental systems contain impenetrable hard spheres. Expressions for $H_{p}$ in a system of impenetrable hard spheres have been established by Torquato and co-workers, ${ }^{46,47}$ but the moments of this function cannot be obtained analytically. Moreover, the scaled transition radius has the highest value for the concentrated samples of the smallest particles PMMA3, which may be the result of colloidal forces. 


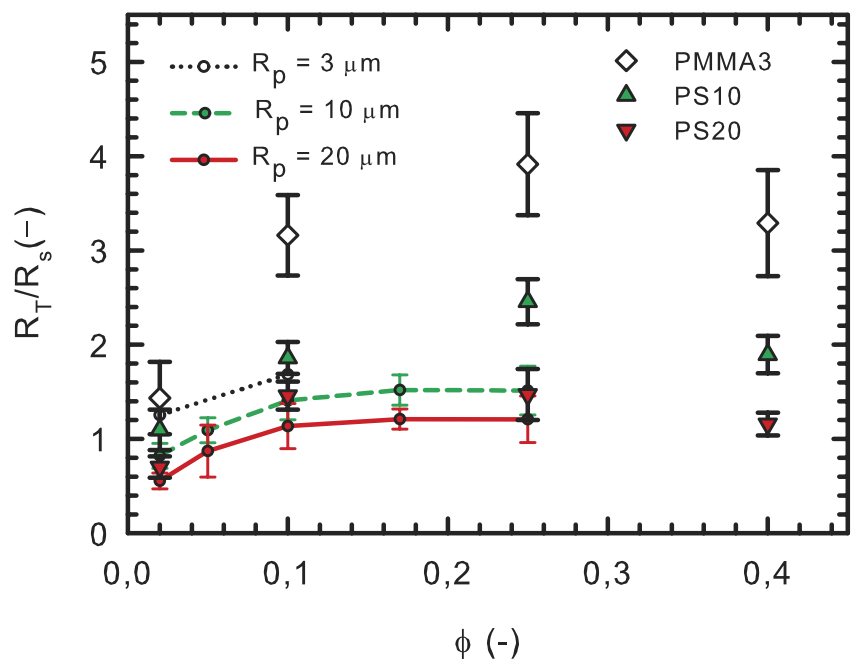

FIG. 8. Overview of the scaled transition radius $R_{T} / R_{S}$ at which the suspension dilution starts for the suspensions with PDMS 2 as medium. The experimental radii are compared to simulations with the same particle size.

\section{Continuous-phase thinning}

As dilution continues, concentration fluctuations eventually lead to particle-free sections in the filament. As discussed in Section IV A, we split this final continuous phase into two regimes: the acceleration regime and the deceleration regime. In the accelerated regime, the filament radius generally thins linearly with time for a short period and the radial velocity $v_{r}=-d R_{m} / d t$ reaches a maximum. We observe that this maximum radial velocity increases for smaller particles and higher particle concentrations. However, this observation does not capture the effect that particle size has on the ultimate breakup time of the suspensions. Although the smallest-particle (PMMA3) suspension has the largest value of $v_{r}$, this suspension takes the longest time to breakup, as demonstrated in Figure 6.

Figure 9 focusses on the final pinch-off dynamics of the three suspensions with fixed volume fraction $\phi=0.25$. The short linear decay where the highest thinning rate is achieved, i.e., the accelerated regime, is demonstrated by a linear fit with a slightly different slope for each sample. For each case, the horizontal dashed line indicates the visible onset of continuous-phase thinning

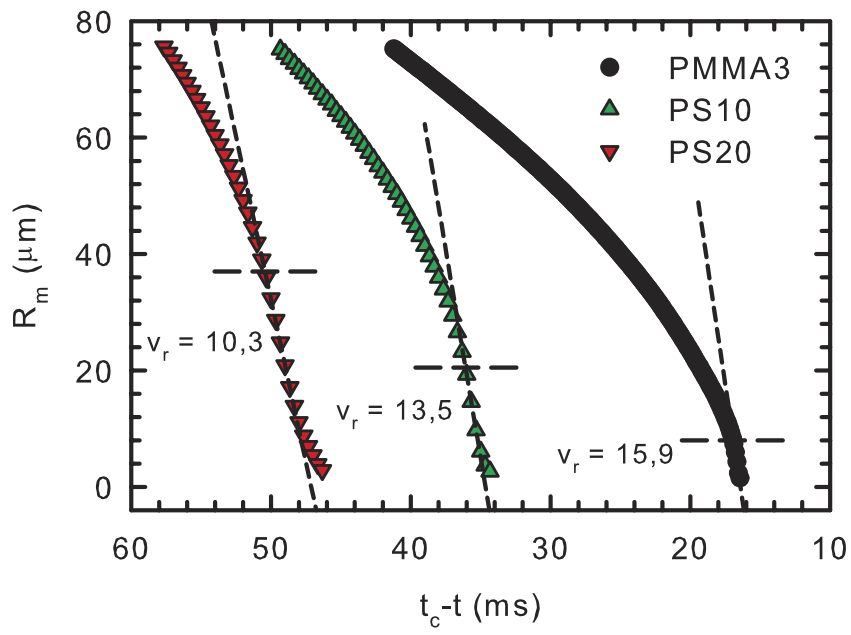

FIG. 9. Plot showing the minimal radius evolution of the same suspensions with $\phi=0.25$ near pinch-off. The increase of the maximum radial velocity with decreasing particle size is shown and the onset of the continuous phase thinning is indicated with a horizontal dashed line. 
between two particle clusters. As this transition occurs at a smaller radius for smaller-particle suspensions, the acceleration regime is limited and large $v_{r}$ cannot affect the dynamics. Consequently, the reduction in breakup time is less than that observed for larger particle sizes. Evidently, it is the concentration fluctuation regime, characterised by the transition radius $R_{T}$, that is key to understanding particulate effects on breakup time, rather than the accelerated regime itself.

As discussed in Section IV A, the acceleration regime is succeeded by a deceleration, which has not been detected in previous experimental studies. The transition into this final regime, and therefore the extent of the deceleration, also depends on the suspension properties in a similar way as previously discussed. For example, in Figure 9, the larger particle cases have a more pronounced deceleration regime, owing to a larger transition radius initiating this final thinning stage. In the PMMA3 case, the final transition is below the spatial and temporal resolution of our setup.

The deceleration regime also exhibits a linear decay (see Figure 4) characterised by a balance between surface tension and the viscosity of the medium fluid. This indicates a return to the self-similar scaling of a viscous Newtonian fluid. This hypothesis is confirmed by scaling the filament profiles near breakup to verify the convergence to the universal Stokes similarity solution $\phi_{S t}$ for the filament radius of a viscous liquid. ${ }^{13,14,17}$ Figure 10 shows the scaled profiles of a suspension of PS20 in PDMS 2 with $\phi=0.10$ near pinch-off. The filament radius $R$ and the axial coordinate $z$ are rescaled as

$$
\begin{gathered}
R^{*}=\frac{R}{R_{m}}, \\
z^{*}=\frac{z-z_{0}}{R_{m}}\left(t_{p}-t\right)^{1-\beta},
\end{gathered}
$$

where $z_{0}$ is axial coordinate corresponding to the minimal radius and $\beta=0.175$ is the scaling exponent for the Stokes similarity solution.

Seven profiles are selected in the final $5 \mathrm{~ms}$ before breakup. The first three scaled profiles, at times 5, 4, and $3 \mathrm{~ms}$ before breakup occur during the accelerated thinning regime and do not exhibit self-similarity. In this regime, a small region around $z^{*}=0$ is observed to be particle free and surrounded by a cluster of particles. However, the last four profiles at times 2, 1.67, 1.33, and $1 \mathrm{~ms}$ before breakup, clearly demonstrate the self-similar nature of the final deceleration regime, approaching the symmetric shape of $\phi_{S t}$. Although the interstitial fluid continues to thin between two particle clusters in this regime, finite-size ceases to affect the thinning dynamics so that the thinning

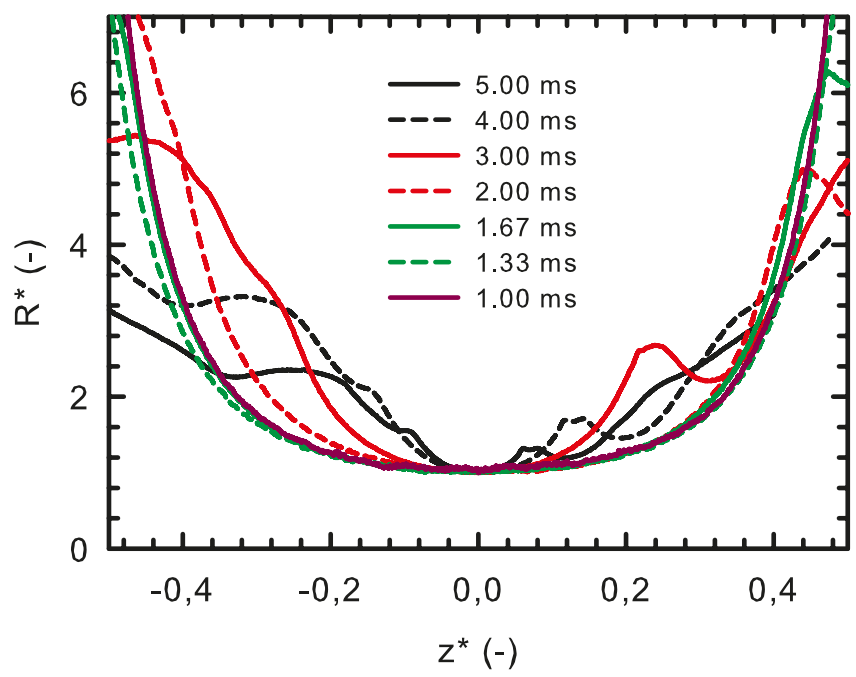

FIG. 10. Scaled experimental filament profiles $R^{*}$ as a function of the scaled axial coordinate $z^{*}$ of a PDMS 2-based suspension of PS20 particles with $\phi=0.10$ near pinch-off. The time until breakup is indicated for each profile. The first three profiles are situated during the accelerated thinning of the continuous phase and the other four are observed in the deceleration stage. The profiles in the latter stage are clearly overlapping and exhibit the same shape as the viscous self-similar solution. 


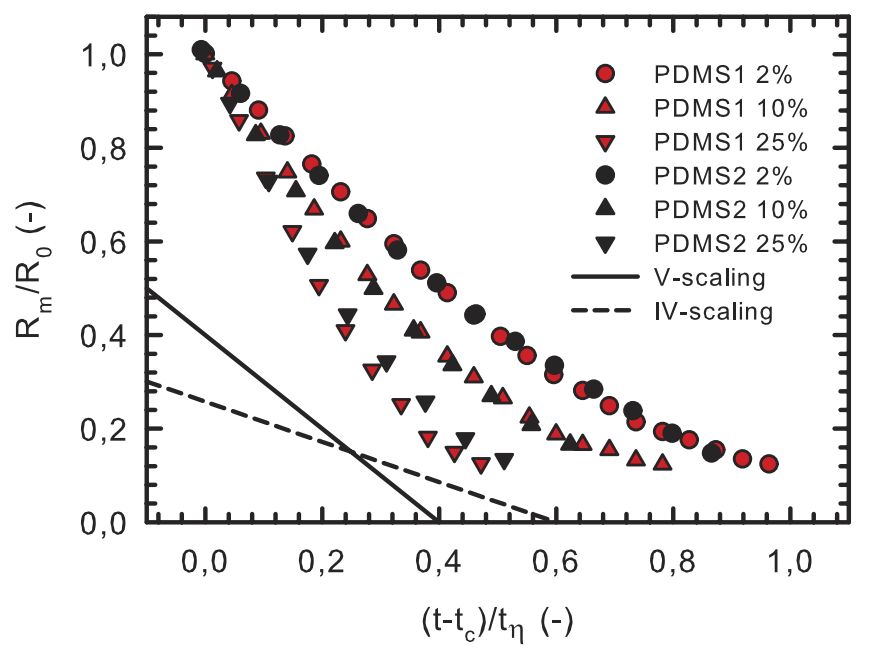

FIG. 11. Deceleration of the filament thinning near pinch-off for a series of suspensions of PS20 with both medium fluids. The time is rescaled with $t_{\eta}$ and minimum filament radius is depicted relative to the particle radius. The deceleration is more pronounced for lower particle volume fractions and for lower medium viscosity, where the thinning dynamics tend to the slower IV scaling before breakup.

rate can be described solely by the Ohnesorge number of the medium fluid. Consequently, we are able to recover the Newtonian thinning transitions described in Section III, as we now discuss.

In Figure 11, we plot the deceleration regime for the PS20 suspension with a range of volume fractions for both medium fluids PDMS 1 and PDMS 2. The deceleration is more prominent for suspensions with a lower particle volume fraction. To allow comparison of samples with different medium viscosities, the time is rescaled with the viscous time scale $t_{\eta}$ (Eq. (15)), where we have chosen the particle radius as the reference radius: $R_{0}=R_{p}$. The time axis is further shifted with $t_{c}$, the time at which filament radius equals the reference radius. The slopes of the two possible self-similar solutions for a viscous liquid are shown in Figure 11. For the suspensions with PDMS 1 as medium fluid, there is the appearance of a transition from the V-scaling to the IV-scaling close to breakup, whereas the PDMS 2-based dispersions persist in following the $\mathrm{V}$-scaling. The switch to the IV-scaling results in a relatively longer deceleration zone for the PDMS 1-based suspensions.

Moreover, the final shape of a Newtonian filament is controlled by the preferred scaling law prior to breakup. ${ }^{2}$ The PDMS 2-based suspensions exhibit a symmetrical pinching, where the breakup occurs in the centre of the filament. In contrast, the symmetry in the filament of the PDMS 1-based suspensions is broken under the influence of inertia, generating a vertical shift of the necking point along the filament towards the particle clusters. This asymmetrical pinch-off results in the formation of a small satellite droplet, which is not experimentally observed with PDMS 2-based suspensions. This contrast between the behaviour of the two medium fluids during the capillary thinning experiments on the pure liquids was noted in Section III. However, the transition to IVthinning in the PDMS 1 suspensions occurs at significantly smaller radii than in the pure liquid, as a consequence of the reference radius $R_{0}$ in Eq. (16) being the onset of the continuous-phase thinning.

\section{CONCLUSION}

We have studied the drop formation of particulate suspensions in a Newtonian medium by investigating the pinch-off dynamics of an unstable liquid bridge. This geometry instantly creates a slender filament enabling the use of self-similar scaling to explain the thinning dynamics. This strategy results in a more straightforward interpretation of the capillary breakup than in previous dripping studies on similar fluids. ${ }^{25,26}$ We have demonstrated excellent agreement between capillary breakup experiments and the model for capillary breakup of particulate suspensions developed by McIlroy and Harlen. ${ }^{36}$ Four thinning regimes are identified during the capillary thinning of suspension. The suspension initially behaves as homogeneous viscous fluid and the filament radius 
decreases linearly in time following the viscous scaling for the effective viscosity of suspension, which is accurately described by the Maron-Pierce model.

As the filament thins, local particle-density fluctuations are amplified resulting in a heterogeneous suspension with diluted zones that exhibit faster thinning rates due to the local decrease in viscosity. We have defined the onset of this concentration fluctuation regime with a transition radius $R_{T}$ that depends on the particle radius and volume fraction in a non-trivial way. A scaling for this transition radius with a structural length scale $R_{S}$ is proposed. This structural length scale is based on the standard deviation of the mean inter particle distance and the relative dependance of the viscosity to small changes of the volume fraction. The value of the transition radius is crucial in estimating the reduction in breakup time compared to a viscous fluid with matching shear viscosity. The local dilution eventually develops a particle-free section, where a small volume of medium fluid is caught between two particle clusters. At this point, the breakup resembles a small-scale filament stretching experiment and the thinning rate reaches a maximum.

Finally, we observe a transition from the accelerated regime to a last deceleration regime, which has until recently ${ }^{48,49}$ not been detected in previous experimental studies. The thinning of the continuous phase appears unaffected by the presence of the particles and follows the viscous scaling of the medium fluid. This scaling has been verified by rescaling the filament profiles to retrieve the symmetric Stokes similarity solution.

\section{ACKNOWLEDGMENTS}

W.M. and C.C. would like to acknowledge financial support from the ERC-2007-StG starting Grant No. 203043 NANOFIB. C.M. and O.G.H. acknowledge support from the EPSRC Reference No. EP/H018913/1.

${ }^{1}$ X. B. Chen, "Modeling and control of fluid dispensing processes: A state-of-the-art review," Int. J. Adv. Manuf. Technol. 43, 276-286 (2009).

${ }^{2}$ C. Clasen, P. M. Phillips, L. Palangetic, and J. Vermant, "Dispensing of rheologically complex fluids: The map of misery," AIChE J. 58, 3242-3255 (2012).

${ }^{3}$ N. F. Morrison and O. G. Harlen, "Viscoelasticity in inkjet printing," Rheol. Acta 49, 619-632 (2010).

${ }^{4}$ J. de Jong, G. de Bruin, H. Reinten, M. van den Berg, H. Wijshoff, M. Versluis, and D. Lohse, "Air entrapment in piezo-driven inkjet printheads," J. Acoust. Soc. Am. 120, 1257-1265 (2006).

${ }^{5}$ O. A. Basaran, H. J. Gao, and P. P. Bhat, "Nonstandard inkjets," Annu. Rev. Fluid Mech. 45, 85-113 (2013).

${ }^{6}$ J. Windle and B. Derby, "Ink jet printing of PZT aqueous ceramic suspensions," J. Mater. Sci. Lett. 18, 87-90 (1999).

${ }^{7}$ B. Derby, "Inkjet printing of functional and structural materials: Fluid property requirements, feature stability, and resolution,” Annu. Rev. Mater. Res. 40, 395-414 (2010).

${ }^{8}$ D. H. Peregrine, G. Shoker, and A. Symon, "The bifurcation of liquid bridges," J. Fluid Mech. 212, 25-39 (1990).

${ }^{9}$ X. D. Shi, M. P. Brenner, and S. R. Nagel, "A cascade of structure in a drop falling from a faucet," Science 265, 219-222 (1994).

${ }^{10}$ X. G. Zhang and O. A. Basaran, “An experimental study of dynamics of drop formation,” Phys. Fluids 7, 1184-1203 (1995).

${ }^{11}$ J. Eggers and T. F. Dupont, "Drop formation in a one-dimensional approximation of the Navier-Stokes equation," J. Fluid Mech. 262, 205-221 (1994).

${ }^{12}$ F. J. Garcia and A. Castellanos, "One-dimensional models for slender axisymmetrical viscous-liquid jets," Phys. Fluids 6 , 2676-2689 (1994).

13 J. Eggers, "Nonlinear dynamics and breakup of free-surface flows," Rev. Mod. Phys. 69, 865-929 (1997).

${ }^{14}$ J. Eggers and E. Villermaux, "Physics of liquid jets," Rep. Prog. Phys. 71, 036601 (2008).

15 J. Eggers, "Universal pinching of 3D axisymmetrical free-surface flow," Phys. Rev. Lett. 71, 3458-3460 (1993).

${ }^{16}$ L. Campo-Deaño and C. Clasen, "The slow retraction method (SRM) for the determination of ultra-short relaxation times in capillary breakup extensional rheometry experiments," J. Non-Newtonian Fluid Mech. 165, 1688-1699 (2010).

${ }^{17}$ D. T. Papageorgiou, "On the breakup of viscous-liquid threads," Phys. Fluids 7, 1529-1544 (1995).

${ }^{18}$ F. J. Galindo-Rosales, M. A. Alves, and M. S. N. Oliveira, "Microdevices for extensional rheometry of low viscosity elastic liquids: A review,” Microfluid. Nanofluid. 14, 1-19 (2013).

${ }^{19}$ G. H. McKinley and A. Tripathi, "How to extract the Newtonian viscosity from capillary breakup measurements in a filament rheometer," J. Rheol. 44, 653-670 (2000).

${ }^{20}$ S. L. Anna and G. H. McKinley, "Elasto-capillary thinning and breakup of model elastic liquids," J. Rheol. 45, 115-138 (2001).

${ }^{21}$ P. Doshi, R. Suryo, O. E. Yildirim, G. H. McKinley, and O. A. Basaran, "Scaling in pinch-off of generalized Newtonian fluids," J. Non-Newtonian Fluid Mech. 113, 1-27 (2003).

${ }^{22}$ G. H. McKinley, "Visco-elasto-capillary thinning and break-up of complex fluids," Rheol. Rev. 3, 1-48 (2005).

${ }^{23}$ M. I. Smith, R. Besseling, M. E. Cates, and V. Bertola, "Dilatancy in the flow and fracture of stretched colloidal suspensions," Nat. Commun. 1, 114 (2010). 
${ }^{24}$ M. Roché, H. Kellay, and H. A. Stone, "Heterogeneity and the role of normal stresses during the extensional thinning of non-Brownian shear-thickening fluids," Phys. Rev. Lett. 107, 134503 (2011).

${ }^{25}$ C. Bonnoit, T. Bertrand, E. Clement, and A. Lindner, "Accelerated drop detachment in granular suspensions," Phys. Fluids 24, 043304 (2012)

${ }^{26}$ R. J. Furbank and J. F. Morris, “An experimental study of particle effects on drop formation,” Phys. Fluids 16, 1777-1790 (2004).

${ }^{27}$ R. J. Furbank and J. F. Morris, "Pendant drop thread dynamics of particle-laden liquids,” Int. J. Multiphase Flow 33, 448-468 (2007).

${ }^{28}$ M. S. Van Deen, T. Bertrand, N. Vu, D. Quéré, E. Clement, and A. Lindner, "Particles accelerate the detachment of viscous liquids," Rheol. Acta 52, 403-412 (2013).

${ }^{29}$ T. Bertrand, C. Bonnoit, E. Clement, and A. Lindner, "Dynamics of drop formation in granular suspensions: The role of volume fraction," Granular Matter 14, 169-174 (2012).

${ }^{30}$ M. Z. Miskin and H. M. Jaeger, "Droplet formation and scaling in dense suspensions," Proc. Natl. Acad. Sci. U. S. A. 109, 4389-4394 (2012)

${ }^{31}$ J. Mewis and N. Wagner, in Colloidal Suspension Rheology, edited by A. Varma (Cambridge University Press, 2012).

${ }^{32}$ I. E. Zarraga, D. A. Hill, and D. T. Leighton, "The characterization of the total stress of concentrated suspensions of noncolloidal spheres in Newtonian fluids," J. Rheol. 44, 185-220 (2000).

${ }^{33}$ S. Maron and P. Pierce, "Application of ree-eyring generalized flow theory to suspensions of spherical particles," J. Colloid Sci. 11, 80-95 (1956).

${ }^{34}$ D. Quemada, "Rheology of concentrated disperse systems and minimum energy-dissipation principle. 1. Viscosityconcentration relationship," Rheol. Acta 16, 82-94 (1977).

${ }^{35}$ I. M. Krieger and T. J. Dougherty, "A mechanism for non-Newtonian flow in suspensions of rigid spheres," Trans. Soc. Rheol. 3, 137-152 (1959).

${ }^{36}$ C. McIlroy and O. G. Harlen, "Modelling capillary breakup of particulate suspensions," Phys. Fluids 26, 033101 (2014).

${ }^{37}$ G. K. Batchelor, "Sedimentation in a dilute dispersion of spheres," J. Fluid Mech. 52, 245-268 (1972).

${ }^{38}$ D. C. Vadillo, W. Mathues, and C. Clasen, "Microsecond relaxation processes in shear and extensional flows of weakly elastic polymer solutions," Rheol. Acta 51, 755-769 (2012).

${ }^{39}$ S. H. Spiegelberg, D. C. Ables, and G. H. McKinley, "The role of end-effects on measurements of extensional viscosity in filament stretching rheometers," J. Non-Newtonian Fluid Mech. 64, 229-267 (1996).

${ }^{40}$ A. Rothert, R. Richter, and I. Rehberg, "Transition from symmetric to asymmetric scaling function before drop pinch-off," Phys. Rev. Lett. 87, 084501 (2001).

${ }^{41}$ J. Eggers, "Drop formation-An overview," Z. Angew. Math. Mech. 85, 400-410 (2005).

${ }^{42}$ M. D. Haw, "Jamming, two-fluid behavior, and 'self-filtration' in concentrated particulate suspensions," Phys. Rev. Lett. 92, 185506 (2004).

${ }^{43}$ R. Suryo and O. A. Basaran, "Local dynamics during pinch-off of liquid threads of power law fluids: Scaling analysis and self-similarity," J. Non-Newtonian Fluid Mech. 138, 134-160 (2006).

${ }^{44}$ S. Torquato, "Statistical description of microstructures," Annu. Rev. Mater. Res. 32, 77-111 (2002).

${ }^{45}$ P. Hertz, "über den gegenseigen durchschnittlichen abstand von punkten, die mit bekannter mittlerer dichte im raume angeordnet sind," Math. Ann. 67, 387-398 (1909).

46 S. Torquato, B. Lu, and J. Rubinstein, "Nearest-neighbor distribution-functions in many-body systems," Phys. Rev. A 41, 2059-2075 (1990).

${ }^{47}$ S. Torquato, "Nearest-neighbor statistics for packings of hard-spheres and disks," Phys. Rev. E 51, 3170-3182 (1995).

${ }^{48}$ A. Lindner, J. E. Fiscina, and C. Wagner, "Single particles accelerate final stages of capillary break-up," Europhys. Lett. 110, 64002 (2015).

${ }^{49}$ H. Zhao, H.-F. Liu, J.-L. Xu, W.-F. Li, and K.-F. Lin, “Inhomogeneity in breakup of suspensions,” Phys. Fluids 27, 063303 (2015). 